MITSUBISHI ELECTRIC RESEARCH LABORATORIES

http://www.merl.com

\title{
Nonbinary LDPC Convolutional Codes for High-Dimensional Modulations
}

\author{
Xia, T.; Koike-Akino, T.; Millar, D.S.; Kojima, K.; Parsons, K.; Miyata, Y.; Sugihara, K.; \\ Matsumoto, W.
}

TR2015-087 July 2015

\begin{abstract}
We show a great potential of nonbinary (NB) LDPC convolutional codes in low- latency decoding for HDM. We show that NB-LDPC can provide considerable performance improvement compared to conventional BICM-ID, which requires soft-decision feedback.
\end{abstract}

2015 Signal Processing in Photonic Communications (SPPCom)

This work may not be copied or reproduced in whole or in part for any commercial purpose. Permission to copy in whole or in part without payment of fee is granted for nonprofit educational and research purposes provided that all such whole or partial copies include the following: a notice that such copying is by permission of Mitsubishi Electric Research Laboratories, Inc.; an acknowledgment of the authors and individual contributions to the work; and all applicable portions of the copyright notice. Copying, reproduction, or republishing for any other purpose shall require a license with payment of fee to Mitsubishi Electric Research Laboratories, Inc. All rights reserved. 



\title{
Nonbinary LDPC Convolutional Codes for High-Dimensional Modulations
}

\author{
Tian Xia ${ }^{1,2}$, Toshiaki Koike-Akino ${ }^{1}$, David S. Millar ${ }^{1}$, Keisuke Kojima $^{1}$, \\ Kieran Parsons $^{1}$, Yoshikuni Miyata $^{3}$, Kenya Sugihara ${ }^{3}$, Wataru Matsumoto ${ }^{3}$ \\ ${ }^{1}$ Mitsubishi Electric Research Labs. (MERL), 201 Broadway, Cambridge, MA 02139, USA. koike@merl.com \\ ${ }^{2}$ School of Electrical Engineering and Computer Science, Louisiana State University, Baton Rouge, LA 70803, USA. \\ ${ }^{3}$ Information Technology R\&D Center, Mitsubishi Electric Corporation, 5-1-1, Ofuna, 247-8501, Japan.
}

\begin{abstract}
We show a great potential of nonbinary (NB) LDPC convolutional codes in lowlatency decoding for HDM. We show that NB-LDPC can provide considerable performance improvement compared to conventional BICM-ID, which requires soft-decision feedback.
\end{abstract}

OCIS codes: (060.4510) Optical communications, (060.1660) Coherent communications, (060.4080) Modulation.

\section{Introduction}

Capacity-achieving low-density parity-check (LDPC) codes have been used for modern optical communications systems [1-8]. Further performance improvement can be provided by introduction of high-dimensional modulation (HDM) [9-15], which directly increases the minimum Euclidean distance by extending the modulation dimension. For HDM, a conventional bit-interleaved coded-modulation (BICM) does not perform well compared to BICM with iterative demodulation (BICM-ID) [15]. However, BICM-ID requires labeling optimization and a soft-decision feedback to the demodulator. Hence, BICM-ID can be less practical due to the high complexity and large latency. By contrast, with nonbinary (NB)-LDPC codes, turbo demodulation is not needed as addressed in [12]. This is a great advantage of NB-LDPC compared to BICM-ID. However, the major obstacle lies in the fact that the decoder complexity increases with the Galois field size. This complexity issue may be mitigated by an introduction of LDPC convolutional codes (CC) with windowed decoding [4,5], as suggested in our theoretical analysis [8]. In this paper, we show a significant performance gain provided by NB-LDPC-CC for HDM in comparison to BICM-ID.

\section{LDPC-coded HDM}

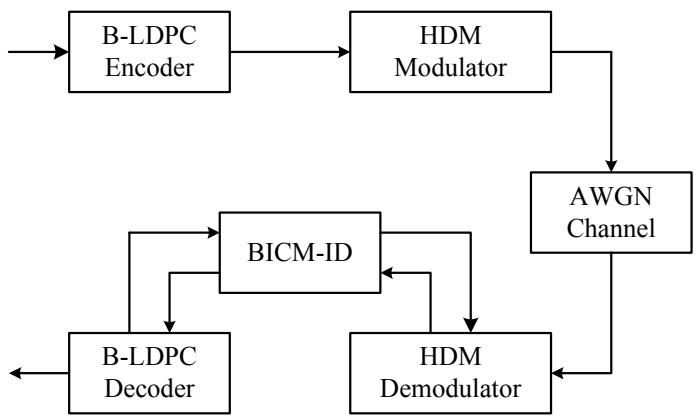

(a) B-LDPC-HDM

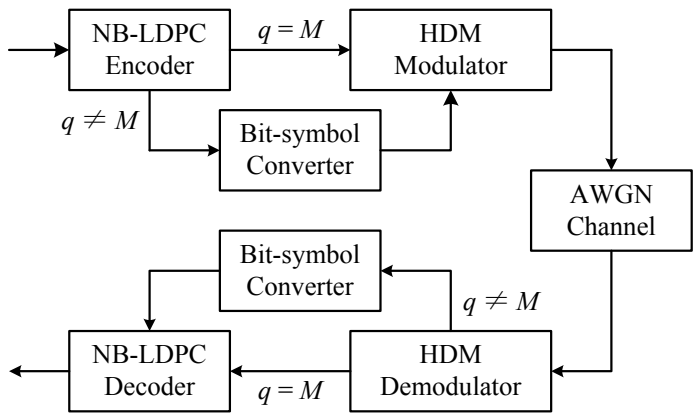

(b) NB-LDPC-HDM

Fig. 1: Schematic of binary LDPC-HDM and NB-LDPC-HDM.

Let $\alpha \mathrm{b} \beta \mathrm{D}$ HDM [13] be defined such that there are $M=2^{\alpha}$ constellation points and each constellation point has coordinates in $\beta$ dimensions. The system diagrams for binary (B) LDPC-HDM and NB-LDPC-HDM schemes are depicted in Fig. 1. For B-LDPC-HDM, at the transmitter side, $\alpha$ consecutive coded bits will be modulated to an $\alpha \mathrm{b} \beta \mathrm{D}$ HDM block; at the receiver side, the HDM demodulator, BICM-ID, and B-LDPC decoder accomplish the iterative demodulation and decoding. Let $N_{\text {iter }}$ be the number of turbo demodulations for BICM-ID. For example with $N_{\text {iter }}=0$, 
there is no feedback from the LDPC decoder to the demodulator, leading to the conventional BICM. Although this feedback mechanism improves the performance for HDM, the decoding latency increases significantly.

For NB-LDPC-HDM, if the Galois field size $q$ is the same as the HDM modulation order $M$, one coded symbol generates one modulated block and the demodulator output can be directly used as input for the NB-LDPC decoder. This motivates us to use NB-LDPC even though a larger Galois field size increases the decoding complexity. When combining convolutional codes with windowed decoding, the complexity issue can be compensated as analyzed in [8].

\section{Performance Results}

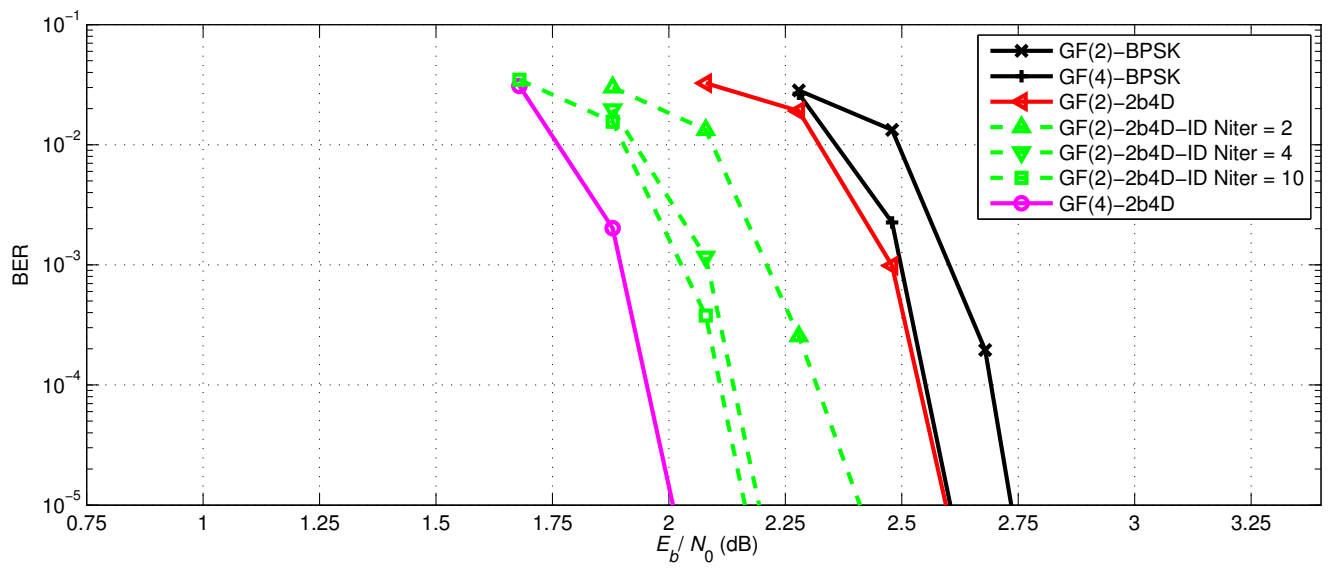

(a) 4-dimensional modulation

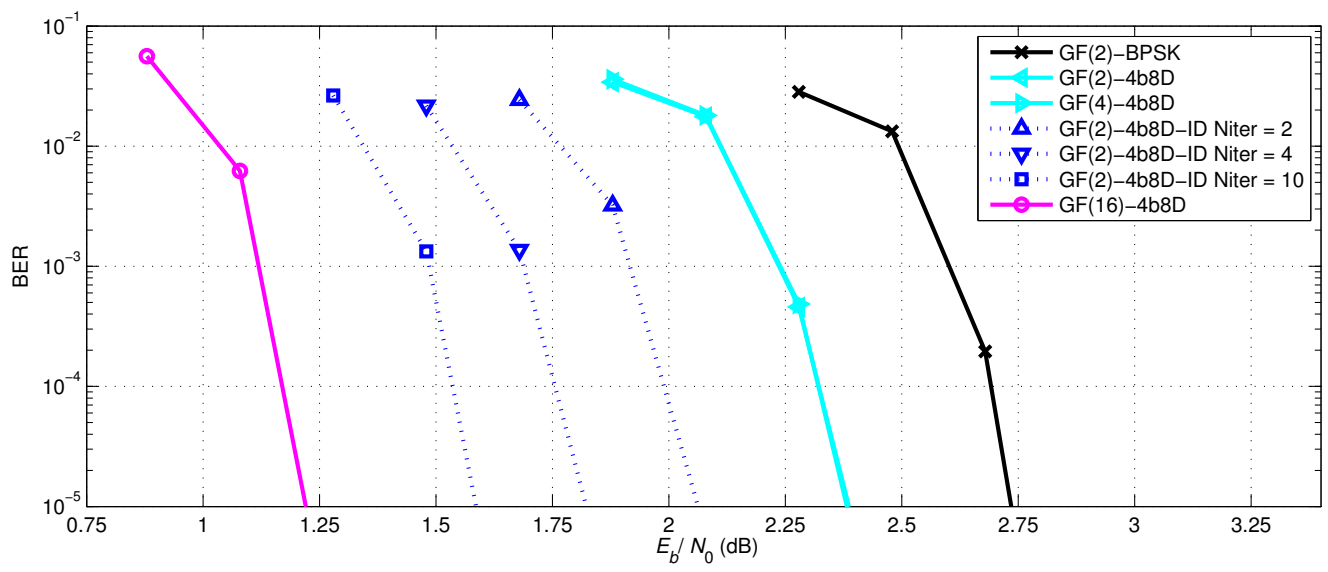

(b) 8-dimensional modulation

Fig. 2: Post-LDPC BER performance for 4D and 8D modulations.

We evaluate the bit-error-rate (BER) performances of binary and nonbinary LDPC codes over different HDM in terms of $E_{\mathrm{b}} / N_{0}$, where $E_{\mathrm{b}}$ is the energy per bit and $N_{0}$ is the noise spectrum density. The BER performance with 1D modulation, i.e., binary phase-shift keying (BPSK), is also evaluated as a reference. We consider the protograph-based LDPC-CC over $\mathbb{G F}(2), \mathbb{G F}(4)$, and $\mathbb{G F}(16)$ with a code rate of 0.78 and a binary codeword length of 38400 . Using quasi-cyclic design technique proposed in [16], we designed a girth-8 $(3,15,20, L)$ LDPC-CC, where check-node degrees are 15 , variable-node degrees are 3 , truncation length is 20 , and graph-lifting factor $L$ is chosen according to the Galois field size $(q=2,4,16)$. We use $2 \mathrm{~b} 4 \mathrm{D}$ and $4 \mathrm{~b} 8 \mathrm{D}$ modulation schemes [13], whose minimum Euclidean distances are, respectively, $1.25 \mathrm{~dB}$ and $3.01 \mathrm{~dB}$ better than the BPSK modulation.

Fig. 2(a) shows the BER performances of binary and nonbinary LDPC codes over $\mathbb{G F}$ (4) with 4D modulation. We can observe the significant benefit by combining NB-LDPC-CC with HDM. By contrast, the NB-LDPC-CC has a very 
marginal gain for 1D modulation. For B-LDPC-HDM, by using turbo demodulation denoted by GF(2)-2b4D-ID, the BER performance can be improved by $0.4 \mathrm{~dB}$. Note that we keep the total number of iterations constant for LDPC decoders when using BICM-ID. It is shown that there is a marginal gain observed when $N_{\text {iter }}$ is increased from 4 to 10 . The NB-LDPC-HDM shows $0.5 \mathrm{~dB}$ gain compared to BICM, and is even better than BICM-ID. Although the decoding complexity is doubled from binary LDPC codes to nonbinary LDPC codes over $\mathbb{G F}(4)$ for fast-Fourier-transform $q$-ary sum-product algorithm (FFT-QSPA), the NB-LDPC-HDM does not need to perform turbo demodulation and windowed decoding can reduce the latency.

We then show BER performance of 8D modulation with LDPC codes over $\mathbb{G F}(2), \mathbb{G F}(4)$, and $\mathbb{G F}(16)$, in Fig. 2(b). It is shown that the $\mathbb{G F}(2)-4 b 8 \mathrm{D}$ and the $\mathrm{GF}(4)-4 \mathrm{~b} 8 \mathrm{D}$ have almost the same BER performance. With BICM-ID ( $\mathbb{G F}(2)-4 b 8 D-I D)$, a $0.8 \mathrm{~dB}$ gain is observed when $N_{\text {iter }}=10$ compared to that of the $\mathbb{G F}(2)-4 b 8 D$. As expected, the $\mathbb{G F}(16)-4 b 8 D$ provides the best performance for $4 \mathrm{~b} 8 \mathrm{D}$ schemes. More than $1 \mathrm{~dB}$ gain is observed for the $\mathrm{GF}(16)-$ $4 \mathrm{~b} 8 \mathrm{D}$ compared to the $\mathrm{GF}(2)-4 \mathrm{~b} 8 \mathrm{D}$. We can also see a significant performance advantage by more than $0.3 \mathrm{~dB}$ over BICM-ID.

\section{Conclusions}

We investigate the combination of nonbinary LDPC convolutional codes (NB-LDPC-CC) with high-dimensional modulation (HDM) schemes. We showed that when the Galois field size is the same as the modulation order, nonbinary LDPC codes with HDM can provide considerable performance improvement, even better than BICM-ID. Although decoder complexity for nonbinary codes increases with larger Galois field size, we do not need soft-decision feedback loop from decoder to demodulator. This feature is a great advantage for hardware implementation. In addition, the use of LDPC convolutional codes can facilitate high-throughput decoder by windowed decoding.

\section{References}

1. I.B. Djordjevic, $O F C$ W3J-4 (2014)

2. S.-Y. Chung, G.D. Forney Jr, T.J. Richardson, R. Urbanke, "On the design of low-density parity-check codes within 0.0045 $\mathrm{dB}$ of the Shannon limit," IEEE COMLET 52 (2001)

3. T.J. Richardson, M.A. Shokrollahi, R.L. Urbanke, "Design of capacity-approaching irregular low-density parity-check codes," IEEE Trans. IT 47 2, (2001)

4. S. Kudekar, T. Richardson, R. Urbanke, "Threshold saturation via spatial coupling: Why convolutional LDPC ensembles perform so well over the BEC," IEEE Trans. IT, 57, 803-834 (2011)

5. L. Schmalen, V. Aref, J. Cho, K. Mahdaviani, ECOC Th.1.3.3 (2014)

6. K. Sugihara, Y. Miyata, T. Sugihara, K. Kubo, H. Yoshida, W. Matsumoto, T. Mizuochi, OFC, OM2B.4 (2013)

7. I. Djordjevic, B. Vasic, "Nonbinary LDPC codes for optical communication systems," IEEE PTL, 17, 2224-2226 (2005)

8. L. Wei, T. Koike-Akino, D.G. Mitchell, T.E. Fuja, D.J. Costello, "Threshold analysis of non-binary spatially-coupled LDPC codes with windowed decoding," ISIT, pp. 881-885 (2014)

9. M. Arabaci, I. Djordjevic, L. Xu, T. Wang, "Four-dimensionalnon binary LDPC-coded modulation schemes for ultra-highspeed optical fiber communication," IEEE PTL, 23, 1280-1282 (2011)

10. E. Agrell, M. Karlsson, "Power-efficient modulation formats in coherent transmission systems," IEEE JLT 27 (2009)

11. L. Beygi, E. Agrell, J.M. Kahn, M. Karlsson, "Rate-adaptive coded modulation for fiber-optic communications," IEEE JLT 32 2, 333-343 (2013)

12. T. Liu, I.B. Djordjevic, "Multidimensional optimal signal constellation sets and symbol mappings for block-interleaved coded-modulation enabling ultra high-speed optical transport," IEEE Photon. J. 64 (2014)

13. D.S. Millar, T. Koike-Akino, S.Ö Arık, K. Kojima, K. Parsons, T. Yoshida, T. Sugihara, "High-dimensional modulation for coherent optical communications systems," Opt. Exp. 227 (2014)

14. A.D. Shiner et al., "Demonstration of an 8-dimensional modulation format with reduced inter-channel nonlinearities in a polarization multiplexed coherent system," Opt. Exp. 2217 (2014)

15. T. Koike-Akino, D.S. Millar, K. Kojima, K. Parsons, OFC W4K.1 (2015)

16. Y. Wang, S. Draper, J. Yedidia, "Hierarchical and high-girth QC LDPC codes," IEEE Trans. IT, 59, 4553-4583 (2013) 